\title{
EQUIVALENT TYPES OF INVARIANT MEANS ON LOCALLY COMPACT GROUPS
}

\author{
P. F. RENAUD
}

Abstract. For $G$ a locally compact amenable group, we establish the equivalence of left invariant means and topologically left invariant means on $L^{\infty}(G)$.

1. Introduction and notation. Let $G$ be a locally compact group with left Haar measure $\mu$. Let $L^{1}(G)$ and $L^{\infty}(G)$ denote the usual Banach function spaces on $G . L^{1}(G)$ is a Banach *-algebra under the convolution operation

and the adjoint map

$$
x * y(g)=\int x(h) y\left(h^{-1} g\right) d \mu(h)
$$

$$
x^{*}(g)=\Delta\left(g^{-1}\right) \overline{x\left(g^{-1}\right)}
$$

where $\Delta$ is the modular function on $G$. A weight on $G$ is a nonnegative function $x \in L^{1}(G)$ such that $\int x(g) d \mu(g)=1$. Denote by $P$ the set of all weights on $G$ and observe that $P$ is a semigroup under convolution. For $f$ a complex-valued function on $G$, define ${ }_{g} f(g \in G)$ by ${ }_{g} f(h)=f\left(g^{-1} h\right)$.

A linear functional $m$ on $L^{\infty}(G)$ is called a mean if

(i) $m(f) \geqq 0$ for all $f \in L^{\infty}(G), f \geqq 0$ and

(ii) $m(1)=1$ where 1 denotes the identity function.

Clearly every weight $x$ in $P$ defines a mean on $L^{\infty}(G)$ via $\langle x, f\rangle=$ $\int x(g) f(g) d \mu(g)$.

Let $m$ be a mean on $L^{\infty}(G) . m$ is called a left invariant mean (LIM) if

$$
m\left({ }_{g} f\right)=m(f) \text { for all } f \in L^{\infty}(G), g \in G .
$$

$m$ is called a topologically left invariant mean (TLIM) if

$$
m(x * f)=m(f) \text { for all } f \in L^{\infty}(G), x \in P,
$$

or equivalently

$$
m(x * f)=m(f) \int x(g) d \mu(g) \text { for all } f \in L^{\infty}(G), x \in L^{1}(G) .
$$

Received by the editors March 10, 1971.

AMS 1970 subject classifications. Primary 43A07; Secondary 22D15, 43A15.

Key words and phrases. Locally compact group, amenable group, invariant means.

(c) American Mathematical Society 1972 
$G$ is called amenable if there exists a LIM on $L^{\infty}(G)$. TLIM's were introduced by Hulanicki [4] as a natural extension of LIM's to nondiscrete groups. Among other results he proved that every TLIM is also a LIM. Subsequently, Namioka [5] showed that the existence of a LIM implies the existence of a TLIM. The purpose of this note is to show that in fact every LIM is also a TLIM. This answers a question raised by Greenleaf (see [3, Lemma 2.2.2 and remarks]).

2. Equivalence of LIM's and TLIM's. We shall now prove the following

THEOREM. Let $G$ be an amenable group, $m$ a LIM on $L^{\infty}(G)$. Then $m$ is a TLIM.

For $f \in L^{\infty}(G)$ and $x \in L^{1}(G)$, left invariance of $m$ gives

$$
m\left(\left({ }_{\sigma} x\right) * f\right)=m\left({ }_{g}(x * f)\right)=m(x * f) \text { for all } g \in G .
$$

Hence $x \rightarrow m(x * f)$ is a left invariant bounded linear functional on $L^{1}(G)$ so that there exists a constant $k(f)$ such that

$$
m(x * f)=k(f) \int x(g) d \mu(g) \text { for all } x \in L^{1}(G) .
$$

It is immediate that $k$ is a mean on $L^{\infty}(G)$. Further if $x \in P$ then $x * x \in P$ so that

$$
k(x * f)=m(x *(x * f))=m((x * x) * f)=k(f)
$$

and $k$ is a TLIM. The theorem will be proved if we show that $m=k$.

Fix $f \in L^{\infty}(G)$. Choose a net $\left\{x_{\gamma}\right\}_{\gamma \in \Omega} \subset P$ such that $w^{*}-\lim _{\gamma} x_{\gamma}=m$ and define $F_{\gamma}$ on $G$ by $\left.F_{\gamma}(g)={ }_{g} x_{\gamma}, f\right\rangle$. Left invariance of $m$ means that $F_{\gamma} \rightarrow$ $m(f)$ pointwise on $G$. The theorem will follow from the following

LEMMA. $\quad F_{\gamma} \rightarrow m(f)$ almost uniformly on every compact subset of $G$.

Proof. If we were dealing with sequences rather than nets, then the lemma would be a trivial application of Egoroff's theorem. With nets, however, a little delicacy is required.

Let $K$ be a compact set with $\mu(K)>0$. For $k$ a positive integer, $\gamma \in \Omega$, define

$$
E_{k, \gamma}=\bigcap_{\gamma^{\prime} \geqq \gamma}\left\{g \in K:\left|F_{\gamma^{\prime}}(g)-m(f)\right| \leqq 1 / k\right\} .
$$

Since $F_{\gamma}$ is continuous, $E_{k, \gamma}$ is a compact subset of $K$. Note that for fixed $k,\left\{E_{k, \gamma}\right\}$ is an increasing net (in the sense that $\gamma \geqq \gamma^{\prime} \Rightarrow E_{k, \gamma} \supseteq E_{k, \gamma^{\prime}}$ ) with $\bigcup_{\gamma} E_{k, \gamma}=K$. Let $\chi_{K}, \chi_{E_{k, \gamma}}$ be the characteristic functions of $K$ and $E_{k, \gamma}$ respectively. We then have that $\left\{\chi_{E_{k, \gamma}}\right\}$ is a bounded monotone increasing net in $L^{\infty}(G)$ for each $k$ and $\chi_{K}=\sup _{\gamma} \chi_{E_{k, \gamma}}$. Now $L^{\infty}(K)$ may be regarded 
as a $W^{*}$-algebra on the Hilbert space $L^{2}(K)$. The predual of $L^{\infty}(K)$ is $L^{1}(K)$ so that every nonnegative element in $L^{1}(K)$ is a normal positive linear functional on $L^{\infty}(K)$ [1, Chapitre 1, $\S \S 3$ and 4]. Hence $\left\langle\chi_{K}, \chi_{K}\right\rangle=$ $\sup _{\gamma}\left\langle\chi_{K}, \chi_{E_{k, \gamma}}\right\rangle$ or,

$$
\lim _{\gamma} \mu\left(E_{k, \gamma}\right)=\mu(K) \text { for each } k .
$$

Fix $\varepsilon>0$. For each $k$, choose $\gamma_{k}$ such that $\mu\left(K \backslash E_{k, \gamma_{k}}\right)<\varepsilon / 2^{k}$ and let $E_{0}=$ $\bigcap_{k} E_{k, \gamma_{k}} . E_{0}$ is a compact set and

$$
\mu\left(K \backslash E_{0}\right)=\mu\left(\bigcup_{k} K \mid E_{k, \gamma_{k}}\right) \leqq \sum_{k} \mu\left(K \backslash E_{k, \gamma_{k}}\right)<\varepsilon .
$$

Finally it is clear that $F_{\gamma} \rightarrow m(f)$ uniformly on $E_{0}$.

It should be noted that the above theorem resembles somewhat the condition (FC*) of [2]. Using a technique similar to the one employed in the proof of Lemma 1.4.3 of [2], we could show directly that the above lemma implies that $F_{\gamma} \rightarrow m(f)$ uniformly on all compact sets.

Proof OF THEOREM. By the above lemma we can find a compact set $E$ with $\mu(E)>0$ such that $F_{\gamma} \rightarrow m(f)$ uniformly on $E$. Therefore

But

$$
\lim _{\gamma} \int_{E} F_{\gamma}(g) d \mu(g)=m(f) \mu(E) .
$$

$$
\begin{aligned}
\int_{E} F_{\gamma}(g) d \mu(g) & =\int_{G} \chi_{E}(g)\left[\int_{G} x_{\gamma}\left(g^{-1} h\right) f(h) d \mu(h)\right] d \mu(g) \\
& =\int_{G}\left(\chi_{E} * x_{\gamma}\right)(h) f(h) d \mu(h) \\
& =\left\langle\chi_{E} * x_{\gamma}, f\right\rangle=\left\langle x_{\gamma}, \chi_{E}^{*} * f\right\rangle
\end{aligned}
$$

so that $\lim _{\gamma} \int_{E} F_{\gamma}(g) d \mu(g)=m\left(\chi_{E}^{*} * f\right)$. Hence

$$
\begin{aligned}
m(f) \mu(E) & =m\left(\chi_{E}^{*} * f\right) \\
& =k(f) \int \chi_{E}^{*}(g) d \mu(g)=k(f) \mu(E) .
\end{aligned}
$$

Therefore $m(f)=k(f)$ and $f$ being arbitrary, $m=k$. Hence $m$ is a TLIM.

The notion of LIM and TLIM may be applied to $C B(G)$ - the space of bounded continuous functions on $G$. The method above may be applied here to show that on $C B(G)$ every LIM is again a TLIM.

\section{REFERENCES}

1. J. Diximier, Les algèbres d'opérateurs dans l'espace Hilbertien, Gauthier-Villars, Paris, 1969.

2. W. R. Emerson and F. P. Greenleaf, Covering properties and Folner conditions for locally compact groups, Math. Z. 102 (1967), 370-384. MR 36 \#3912. 
3. F. P. Greenleaf, Invariant means on topological groups and their applications, Van Nostrand Math. Studies, no. 16, Van Nostrand, New York, 1969. MR 40 \#4776.

4. A. Hulanicki, Means and Følner condition on locally compact groups, Studia Math. 27 (1966), 87-104. MR 33 \#4178.

5. I. Namioka, On a recent theorem by H. Reiter, Proc. Amer. Math. Soc. 17 (1966), 1101-1102. MR 34 \#4406

Department of Mathematics, University of Canterbury, Christchurch, New Zealand 